\title{
Performance Analysis of CDMA Signature Optimization with Finite Rate Feedback
}

\author{
Wei Dai ${ }^{\dagger}$, Youjian Liu $^{\dagger}$, Brian Rider ${ }^{\ddagger}$ \\ ${ }^{\dagger}$ Dept. of Electrical and Computer Eng., ${ }^{\ddagger}$ Math Department \\ University of Colorado at Boulder \\ Boulder CO 80303, USA \\ Email: dai@colorado.edu, eugeneliu@ieee.org, brider@euclid.colorado.edu
}

\begin{abstract}
We analyze the performance of CDMA signature optimization with finite rate feedback. For a particular user, the receiver selects a signature vector from a signature codebook to avoid the interference from other users, and feeds the corresponding index back to this user through a finite rate and error-free feedback link. We assume the codebook is randomly constructed where the entries are independent and isotropically distributed. It has been shown that the randomly constructed codebook is asymptotically optimal. In this paper, we consider two types of signature selection criteria. One is to select the signature vector that minimizes the interference from other users. The other one is to select the signature vector to match the weakest interference directions. By letting the processing gain, number of users and feedback bits approach infinity with fixed ratios, we derive the exact asymptotic formulas to calculate the average interference for both criteria. Our simulations demonstrate the theoretical formulas. The analysis can be extended to evaluate the signalto-interference plus noise ratio performance for both match filter and linear minimum mean-square error receivers.
\end{abstract}

\section{INTRODUCTION}

In a direct-sequence code-division multiple access (DSCDMA) system, the performance is mainly limited by the interference among users. To minimize the interference, every particular user wants to select a signature vector from a signature codebook to avoid the interference from other users. In this paper, we assume that the receiver (base station) has the perfect information of the signatures. It selects a signature for a particular user according to some criterion, and feeds the corresponding index to this user through a feedback link. We also assume that the feedback link is error-free and rate limited. Due to the finite feedback rate, there is a performance degradation compared to the infinite feedback rate case. We are interested in quantifying the effect of finite rate feedback.

This problem has been studied in [1]. A randomly constructed signature codebook is assumed in [1] where the codebook entries are independent and isotropically distributed. The interference signature matrix is assumed to have independent and identically distributed (i.i.d.) Gaussian elements. A particular user chooses the signature vector from the signature codebook to maximize signal-to-interference plus noise ratio (SINR). For the matched filter receiver, this criterion is equivalent to select the signature to minimize the interference. In [1], an asymptotic lower bound is given on the average interference.
The main contribution of this paper is to derive the exact performance limit. In this paper, we use the average interference as a performance measure, which is independent of specific receivers and applications. We consider two signature selection criteria. One is to minimize the interference from other users, same as the one in [1]. The other one is more intuitive. We select the signature vector to match the weakest interference directions, or equivalently, to be as orthogonal as possible to the strong interference directions. To analyze the corresponding performance, we let the processing gain, number of users and feedback bits approach infinity simultaneously with fixed ratios. By asymptotic analysis, we derive lower bounds and upper bounds on the average interference for both criteria. For each criteria, the asymptotic upper bound meets the asymptotic lower bound. Therefore, these bounds provide the exact performance limit. The corresponding analysis can be extended to evaluate the SINR performance for both match filter and linear minimum mean-square error (MMSE) receivers.

\section{SySTEM MOdeL}

In a sampled discrete-time symbol-synchronous DS-CDMA system, the received vector can be written as

$$
\mathbf{Y}=\sum_{j=1}^{m} B_{j} \mathbf{s}_{j}+\mathbf{W},
$$

where $B_{j} \in \mathbb{C}$ and $\mathbf{s}_{j} \in \mathbb{C}^{n \times 1}$ are the transmitted symbol and the signature vector for user $j$ respectively, and $\mathbf{W} \in \mathbb{C}^{n \times 1}$ is the additive white Gaussian noise vector with zero mean and covariance matrix $\sigma^{2} \mathbf{I}$. The processing gain (length of the signature vector) is $n$, and $m$ is the number of users. We also assume that the transmitted symbols $B_{j}$ 's are independent and with the same power (variance) 1.

We assume that the receiver has perfect knowledge about the signature vectors $\mathbf{s}_{j}$ 's. For a particular user, without loss of generality, user 1 is assumed, the receiver selects his signature to avoid the interference from the other users. It feeds the corresponding index back to user 1 through a finite rate and error-free feedback link. The rate of the feedback link is assumed to be up to $R_{\mathrm{fb}}$ bits. In order to accomplish this, a 
signature codebook $\mathcal{B}$ with size $2^{R_{\mathrm{fb}}}$ is declared to both the receiver and user 1 .

We assume that the signature codebook $\mathcal{B}$ is randomly constructed. Specifically, $\mathcal{B}=\left\{\mathbf{v}_{1}, \cdots, \mathbf{v}_{2} R_{\mathrm{fb}}\right\}$, where $\mathbf{v}_{k}=$ $\mathbf{z}_{k} /\left\|\mathbf{z}_{k}\right\|, \mathbf{z}_{k}=\left[z_{1, k}, \cdots, z_{n, k}\right]$ and $z_{i, k}$ are i.i.d. $\mathcal{C N}(0,1)$ for all $1 \leq i \leq n$ and $1 \leq k \leq 2^{R_{\mathrm{fb}}}$. In this way, it is guaranteed that $\mathbf{v}_{k}$ 's are independent and isotropically distributed unitary complex vectors. It has been shown that the randomly constructed codebook is asymptotically optimal [1], [2].

In this paper, we use the average interference as the performance measure. Let $\mathbf{S} \in \mathbb{C}^{n \times(m-1)}$ be the interference matrix for user 1, whose columns are the interfering signatures $\mathbf{s}_{2}, \cdots, \mathbf{s}_{m}$. We assume that $\mathbf{S}$ has i.i.d. complex Gaussian entries with zero mean and variance $\frac{1}{n}$, same as the assumption in $[1]^{1}$. For a given interference matrix $\mathbf{S}$, the interference to user 1 is defined by

$$
I_{\mathbf{S}} \triangleq \sum_{j=2}^{m}\left|\left\langle\mathbf{s}_{1}, \mathbf{s}_{j}\right\rangle\right|^{2}=\mathbf{s}_{1}^{\dagger} \mathbf{S S}^{\dagger} \mathbf{s}_{1} .
$$

The average interference is defined by $I \triangleq \mathrm{E}_{\mathbf{S}}\left[\mathrm{E}_{\mathcal{B}}\left[I_{\mathbf{S}}\right]\right]$.

In this paper, we consider two types of signature selection criteria. The first one is to minimize the interference from other users, i.e.,

$$
\mathbf{s}_{1}=\underset{\mathbf{v}_{k} \in \mathcal{B}}{\arg \min } \mathbf{v}_{k}^{\dagger} \mathbf{S S}^{\dagger} \mathbf{v}_{k}
$$

The second one is to select the signature vector to match the weakest interference directions (or equivalently, to be as orthogonal as possible to the strong interference directions). Let $d$ be the multiplicity of the smallest singular value of $\mathbf{S}$. Let $\mathbf{u}_{n-d+1}, \mathbf{u}_{n-d+2}, \cdots, \mathbf{u}_{n}$ be the $d$ left singular vectors of $\mathbf{S}$ corresponding to the smallest singular value and $\mathbf{U}_{d}=$ $\left[\mathbf{u}_{n-d+1} \cdots \mathbf{u}_{n}\right]$. The direction matching criterion is

$$
\mathbf{s}_{1}=\underset{\mathbf{v}_{k} \in \mathcal{B}}{\arg \max } \mathbf{v}_{k}^{\dagger} \mathbf{U}_{d} \mathbf{U}_{d}^{\dagger} \mathbf{v}_{k}
$$

For both criteria, we shall derive the asymptotic performance limit in Sections III and IV respectively. The corresponding analysis can be extended to SINR performance evaluation for both match filter and linear MMSE receivers [2].

\section{ANALysis FOR INTERFERENCE Minimization}

This section is devoted to calculate the average interference for the interference minimization criterion in (1). By letting the processing gain, number of users and feedback bits approach infinity simultaneously with fixed ratios, we derive the exact performance limit. The result is given in Theorem 1

\footnotetext{
${ }^{1}$ It is more natural to assume that the columns in $\mathbf{S}$ are independent and isotropically distributed unitary complex vectors. However, the asymptotic statistics of $\mathbf{S}$ are the same for both assumptions. We adopt the assumption in [1] for fair comparison. In Section $\nabla$ we shall show that the difference between these two assumptions is indistinguishable for relatively large systems.
}

Theorem 1: Define

$$
d \mu_{\lambda} \triangleq\left\{\begin{array}{rr}
\frac{\sqrt{\left(\lambda^{+}-\lambda\right)\left(\lambda-\lambda^{-}\right)}}{2 \pi \lambda} \mathbb{1}_{\left[\lambda^{-}, \lambda^{+}\right]} d \lambda & \text { if } n \leq m \\
{\left[\frac{1}{\tau} \frac{\sqrt{\left(\lambda^{+}-\lambda\right)\left(\lambda-\lambda^{-}\right)}}{2 \pi \lambda} \mathbb{1}_{\left[\lambda^{-}, \lambda^{+}\right]}\right.} & \\
\left.+\frac{\tau-1}{\tau} \delta(\lambda)\right] d \lambda & \text { if } n>m
\end{array}\right.
$$

for a $\tau \geq 1$, where $\lambda^{ \pm}=(1 \pm \sqrt{\tau})^{2}$. For convenience, define $\lambda_{t}^{-} \triangleq \lambda^{-}$if $n \leq m$ and $\lambda_{t}^{-} \triangleq 0$ if $n>m$. For any $x \in$ $\left(\lambda_{t}^{-}, \lambda^{+}\right)$and $\alpha \in\left[0, \frac{1}{x-\lambda_{t}^{-}}\right]$, define

$$
\psi(x, \alpha) \triangleq \int_{\lambda_{t}^{-}}^{\lambda^{+}} \log (1+\alpha(\lambda-x)) d \mu_{\lambda}
$$

and

$$
\bar{\psi}(x) \triangleq \max _{\alpha \in\left[0, \frac{1}{x-\lambda_{t}^{-}}\right]} \psi(x, \alpha) .
$$

Let $n, m$ and $R_{\mathrm{fb}}$ approach infinity simultaneously with fixed ratios $\tau=\max (n, m) / \min (n, m), \bar{r}=\min (n, m) / n$ and $c=R_{\mathrm{fb}} / n$. For any $0<c<\infty$, there exists an $x_{c} \in\left(\lambda_{t}^{-}, \lambda^{+}\right)$ such that $c \log 2=\bar{\psi}\left(x_{c}\right)$ and

$$
\lim _{\left(n, m, R_{\mathrm{fb}}\right) \rightarrow \infty} I^{(n)}=\bar{r} x_{c} .
$$

Remark 1: Theorem 11 is only valid when $0<c=R_{\mathrm{fb}} / n<$ $\infty$. However, it also provides the exact performance limit when $c \rightarrow 0^{+}$or $c \rightarrow+\infty$. Elementary computations show that as $c \rightarrow 0^{+}, \bar{r} x_{c} \rightarrow \bar{r} \bar{\lambda} \triangleq \bar{r} \int_{\lambda_{t}^{-}}^{\lambda^{+}} \lambda d \mu_{\lambda}$ the average eigenvalue, and as $c \rightarrow+\infty, \bar{r} x_{c} \rightarrow \bar{r} \lambda_{t}^{-}$the minimum eigenvalue, which are consistent with intuition.

The essential idea behind Theorem 1 is the same as that behind the standard large deviation technique. The lower bound is derived by Chebyshev's inequality and the upper bound is derived by the twisted distribution. Similar to the result in large deviation technique, the asymptotic lower and upper bounds are identical. We shall outline the proofs for the lower and upper

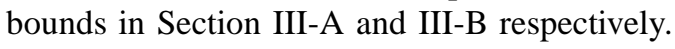

As a beginning, we express the average interference in a convenient form. For a CDMA system with finite $n$ and $m$, the average interference is given by

$$
I^{(n)}=\mathrm{E}_{\mathbf{S}}\left[\mathrm{E}_{\mathcal{B}}\left[\min \mathbf{v}_{k}^{\dagger} \mathbf{S} \mathbf{S}^{\dagger} \mathbf{v}_{k} \mid \mathbf{S}\right]\right]
$$

where $I^{(n)}$ is used to emphasize that they are for finite $n$ and $m$. Let $\mathbf{H}_{n}$ be an $n \times m$ matrix whose entries are i.i.d. complex Gaussian $\mathcal{C N}(0,1)$. Obviously, the statistics of $\mathbf{S}$ is the same as $\frac{1}{\sqrt{n}} \mathbf{H}_{n}$. Therefore, $\mathbf{S S}^{\dagger}=\frac{r}{n} \frac{1}{r} \mathbf{H}_{n} \mathbf{H}_{n}^{\dagger}$ where $r \triangleq \min (n, m)$. 
Let $\lambda_{i}$ be the $i^{\text {th }}$ eigenvalue of the matrix $\frac{1}{r} \mathbf{H}_{n} \mathbf{H}_{n}^{\dagger}$. We have

$$
\begin{aligned}
I^{(n)} & \stackrel{(a)}{=} \mathrm{E}_{\mathbf{S}}\left[\mathrm{E}_{\mathcal{B}}\left[\min _{k} \frac{\mathbf{z}_{k}^{\dagger} \mathbf{S S}^{\dagger} \mathbf{z}_{k}}{\left\|\mathbf{z}_{k}\right\|^{2}} \mid \mathbf{S}\right]\right] \\
& =\frac{r}{n} \mathrm{E}_{\mathbf{H}_{n}}\left[\mathrm{E}_{\mathcal{B}}\left[\min _{k} \frac{\mathbf{z}_{k}^{\dagger} \frac{1}{r} \mathbf{H}_{n} \mathbf{H}_{n}^{\dagger} \mathbf{z}_{k}}{\left\|\mathbf{z}_{k}\right\|^{2}} \mid \mathbf{H}_{n}\right]\right] \\
& \stackrel{(b)}{=} \frac{r}{n} \mathrm{E}_{\mathbf{H}_{n}}\left[\mathrm{E}_{\mathcal{B}}\left[\min _{k} \frac{\mathbf{z}_{k}^{\dagger} \mathbf{U} \mathbf{\Lambda} \mathbf{U}^{\dagger} \mathbf{z}_{k}}{\left\|\mathbf{z}_{k}\right\|^{2}} \mid \mathbf{H}_{n}\right]\right] \\
& \stackrel{(c)}{=} \frac{r}{n} \mathrm{E}_{\boldsymbol{\lambda}}\left[\mathrm{E}_{\mathcal{B}}\left[\min _{k} \frac{\sum_{i=1}^{n} \lambda_{i}\left|z_{i, k}\right|^{2}}{\sum_{i=1}^{n}\left|z_{i, k}\right|^{2}} \mid \boldsymbol{\lambda}\right]\right],
\end{aligned}
$$

where

(a) follows from the random construction of the signature codebook $\mathcal{B}$,

(b) follows from the singular value decomposition of $\frac{1}{r} \mathbf{H}_{n} \mathbf{H}_{n}^{\dagger}$, and

(c) follows from the fact that $\mathbf{z}_{k}$ and $\mathbf{U} \mathbf{z}_{k}$ are statistically equal for any $n \times n$ unitary matrix $\mathbf{U}$ [3].

Note that given $\boldsymbol{\lambda}$, the random variables $\sum_{i=1}^{n} \lambda_{i}\left|z_{i, k}\right|^{2} / \sum_{i=1}^{n}\left|z_{i, k}\right|^{2}, 1 \leq k \leq 2^{R_{\mathrm{fb}}}$, are i.i.d.. Denote the corresponding conditional distribution function by $F_{n}(x \mid \boldsymbol{\lambda})$, then

$$
\begin{aligned}
F_{n}(x \mid \boldsymbol{\lambda}) & =\operatorname{Pr}\left(\frac{\sum_{i=1}^{n} \lambda_{i}\left|z_{i}\right|^{2}}{\sum_{i=1}^{n}\left|z_{i}\right|^{2}} \leq x \mid \boldsymbol{\lambda}\right) \\
& =\operatorname{Pr}\left(\sum_{i=1}^{n}\left(\lambda_{i}-x\right)\left|z_{i}\right|^{2} \leq 0 \mid \boldsymbol{\lambda}\right) .
\end{aligned}
$$

It is worthy to keep in mind that this distribution function is function of $\lambda$. Due to the independence of $\sum_{i=1}^{n} \lambda_{i}\left|z_{i, k}\right|^{2} / \sum_{i=1}^{n}\left|z_{i, k}\right|^{2}, 1 \leq k \leq 2^{R_{\mathrm{fb}}}$, for a given $\lambda$, we have

$\operatorname{Pr}\left(\min _{k} \frac{\sum_{i=1}^{n} \lambda_{i}\left|z_{i, k}\right|^{2}}{\sum_{i=1}^{n}\left|z_{i, k}\right|^{2}} \leq x \mid \boldsymbol{\lambda}\right)=1-\left(1-F_{n}(x \mid \boldsymbol{\lambda})\right)^{2^{R_{\mathrm{fb}}}}$.

Therefore,

$$
\begin{aligned}
& \mathrm{E}_{\mathcal{B}}\left[\min \frac{\sum_{i=1}^{n} \lambda_{i}\left|z_{j}\right|^{2}}{\sum_{i=1}^{n}\left|z_{j}\right|^{2}} \mid \boldsymbol{\lambda}\right] \\
& =\int x \cdot d\left[1-\left(1-F_{n}(x \mid \boldsymbol{\lambda})\right)^{2^{R_{\mathrm{fb}}}}\right] \\
& =\lambda_{\min }+\int_{\lambda_{\min }}^{\lambda_{\max }}\left(1-F_{n}(x \mid \boldsymbol{\lambda})\right)^{2^{R_{\mathrm{fb}}}} d x
\end{aligned}
$$

and

$$
I^{(n)}=\frac{r}{n} \mathrm{E}_{\boldsymbol{\lambda}}\left[\lambda_{\min }+\int_{\lambda_{\min }}^{\lambda_{\max }}\left(1-F_{n}(x \mid \boldsymbol{\lambda})\right)^{2^{R_{\mathrm{fb}}}} d x\right],
$$

where $\lambda_{\min }$ and $\lambda_{\max }$ are the minimum and maximum eigenvalues of $\frac{1}{r} \mathbf{H}_{n} \mathbf{H}_{n}^{\dagger}$ respectively.

It is difficult to calculate (5) for finite $n$ and $m$. We let $n$, $m$ and $R_{\mathrm{fb}}$ approach infinity with fixed ratios and derive lower and upper bounds on $I$ for large systems.

\section{A. The Asymptotic Lower Bound}

The following lemma provides an asymptotic lower bound on the average interference.

Lemma 1: Following the definitions in Theorem 1 let $n, m$ and $R_{\mathrm{fb}}$ approach infinity simultaneously with fixed ratios $\tau, \bar{r}$ and $c$. For any $0<c<\infty$,

$$
\lim _{\left(n, m, R_{\mathrm{fb}}\right) \rightarrow \infty} I^{(n)} \geq \bar{r} x_{c} .
$$

Due to the length limit, we only sketch the proof. It is based on Chebyshev's inequality and the asymptotic behavior of the spectrum of a Wishart matrix. Recall that we are dealing with a distribution function conditioned on the random vector $\boldsymbol{\lambda}$, we need to define some "good" set of $\boldsymbol{\lambda}$, say $A_{\lambda}^{n}$, which will appear soon. By Chebyshev's inequality, it can be proved that

$$
\begin{aligned}
F_{n}(x \mid \boldsymbol{\lambda}) & =\operatorname{Pr}\left(\sum_{i=1}^{n}\left(\lambda_{i}-x\right)\left|z_{i}\right|^{2} \leq 0 \mid \boldsymbol{\lambda}\right) \\
& \leq \frac{1}{e^{-\alpha \cdot 0}} \int e^{-\alpha \sum\left(\lambda_{i}-x\right)\left|z_{i}\right|^{2}} d \mu_{\mathbf{z}} \\
& =\exp \left(-\sum_{i=1}^{n} \log \left(1+\alpha\left(\lambda_{i}-x\right)\right)\right)
\end{aligned}
$$

for $\forall \alpha \in\left(0, \frac{1}{x-\lambda_{t}^{-}}\right)$and $\boldsymbol{\lambda} \in A_{\lambda}^{n}$, where the set $A_{\lambda}^{n}$ is defined by

$$
\begin{gathered}
A_{\boldsymbol{\lambda}}^{n} \triangleq\left\{\boldsymbol{\lambda}:\left|\psi_{n}(\boldsymbol{\lambda}, x, \alpha)-\psi(x, \alpha)\right| \leq \epsilon_{1}\right\} \\
\cap\left\{\boldsymbol{\lambda}:\left|\lambda_{\min }-\lambda_{t}^{-}\right| \leq \epsilon_{2}\right\}, \\
\psi_{n}(\boldsymbol{\lambda}, x, \alpha) \triangleq \frac{1}{n} \sum_{i=1}^{n} \log \left(1+\alpha\left(\lambda_{i}-x\right)\right),
\end{gathered}
$$

and the positive numbers $\epsilon_{1}$ and $\epsilon_{2}$ are small enough. By the asymptotic behavior of the spectrum of a Wishart matrix, it can be shown that $\operatorname{Pr}\left(A_{\lambda}^{n}\right) \rightarrow 1$.

Now take a small $\epsilon>0$ such that $x_{c}-\epsilon>\lambda_{t}^{-}$. Let $x=x_{c}-\epsilon$. It can be proved that we can always find an $\alpha \in\left(0, \frac{1}{x-\lambda_{t}^{-}}\right)$ such that $\psi(x, \alpha)>c \log 2$. Then for $\forall \delta>0$,

$$
\begin{aligned}
& \left(1-F_{n}(x \mid \boldsymbol{\lambda})\right)^{2^{c n}} \\
& \geq\left(1-e^{-n \frac{1}{n} \sum \log \left(1+\alpha\left(\lambda_{i}-x\right)\right)}\right)^{2^{c n}} \\
& =\exp \left(2^{c n} \log \left(1-e^{-n \frac{1}{n} \sum \log \left(1+\alpha\left(\lambda_{i}-x\right)\right)}\right)\right) \\
& \stackrel{(a)}{\geq} \exp \left(-e^{-n\left[\psi(x, \alpha)-\epsilon_{1}-c \log 2\right]}(1+O(1))\right) \\
& \stackrel{(b)}{\geq} 1-\delta
\end{aligned}
$$

on $A_{\lambda}^{n}$ for $n$ large enough, where

(a) follows by Taylor series expansion, and

(b) follows from the fact that we are able to choose $\epsilon_{1}>0$ small enough such that $\psi(x, \alpha)-\epsilon_{1}-c \log 2>0$. 
Then

$$
\begin{aligned}
& \mathrm{E}_{\boldsymbol{\lambda}}\left[\int_{\lambda_{\min }}^{\lambda_{\max }}\left(1-F_{n}(x \mid \boldsymbol{\lambda})\right)^{2^{c n}} d x\right] \\
& \stackrel{(c)}{\geq} \mathrm{E}_{\boldsymbol{\lambda}}\left[\int_{\lambda_{t}^{-}+\epsilon_{2}}^{x_{c}-\epsilon}\left(1-F_{n}(x \mid \boldsymbol{\lambda})\right)^{2^{c n}} d x, A_{\boldsymbol{\lambda}}^{n}\right] \\
& \stackrel{(d)}{\geq} \mathrm{E}_{\boldsymbol{\lambda}}\left[\int_{\lambda_{t}^{-}+\epsilon_{2}}^{x_{c}-\epsilon}(1-\delta) d x, A_{\boldsymbol{\lambda}}^{n}\right] \\
& =(1-\delta)\left(x_{c}-\lambda_{t}^{-}-\epsilon-\epsilon_{2}\right) \cdot \operatorname{Pr}\left(A_{\boldsymbol{\lambda}}^{n}\right) \\
& \rightarrow(1-\delta)\left(x_{c}-\lambda_{t}^{-}-\epsilon-\epsilon_{2}\right),
\end{aligned}
$$

where

(c) follows by reducing the integration domain of a nonnegative function, and

(d) follows from (7) and the fact that $F_{n}(x \mid \boldsymbol{\lambda})$ is a nondecreasing function in $x$.

Therefore,

$$
\begin{aligned}
\lim I^{(n)} & =\bar{r} \lim \mathrm{E}_{\boldsymbol{\lambda}}\left[\lambda_{\min }+\int_{\lambda_{\min }}^{\lambda_{\max }}\left(1-F_{n}(x \mid \boldsymbol{\lambda})\right)^{2^{c n}} d x\right] \\
& \geq \bar{r}\left[\lambda_{t}^{-}+(1-\delta)\left(x_{c}-\lambda_{t}^{-}-\epsilon-\epsilon_{2}\right)\right] .
\end{aligned}
$$

By taking $\delta, \epsilon$ and $\epsilon_{2}$ arbitrarily small, we have $\lim I^{(n)} \geq \bar{r} x_{c}$.

\section{B. The Asymptotic Upper Bound}

For the interference minimization criterion in (1), the asymptotic upper bound on the average interference is given in Lemma 2

Lemma 2: Following the definitions in Theorem 11 let $n, m$ and $R_{\mathrm{fb}}$ approach infinity simultaneously with fixed ratios $\tau, \bar{r}$ and $c$. For any $0<c<\infty$,

$$
\lim _{\left(n, m, R_{\mathrm{fb}}\right) \rightarrow \infty} I^{(n)} \leq \bar{r} x_{c} .
$$

Due to the length limit, we omit the detailed proof. A sketch of the proof is given in the below.

To prove the upper bound, roughly speaking, it is sufficient to show that for $\forall \epsilon>0$ and $\forall \delta>0$, if $n$ is large enough, we can upper bound $\left(1-F_{n}(x \mid \boldsymbol{\lambda})\right)^{2^{c n}}$ uniformly by $\delta$, i.e.,

$$
\left(1-F_{n}(x \mid \boldsymbol{\lambda})\right)^{2^{c n}}<\delta \text { for all } x>x_{c}+\epsilon,
$$

on the "good" set $A_{\lambda}^{n}$ (6). Since $\operatorname{Pr}\left(A_{\lambda}^{n}\right) \rightarrow 1$,

$$
\begin{gathered}
I^{(n)=} \mathrm{E}_{\boldsymbol{\lambda}}\left[\lambda_{\min }+\int_{\lambda_{\min }}^{\lambda_{\max }}\left(1-F_{n}(x \mid \boldsymbol{\lambda})\right)^{2^{R_{\mathrm{fb}}}} d x\right] \\
\leq \bar{r}\left\{\mathrm{E}_{\boldsymbol{\lambda}}\left[\lambda_{\min }\right]+\mathrm{E}_{\boldsymbol{\lambda}}\left[\int_{\lambda_{\min }}^{x_{c}+\epsilon} 1 d x\right]\right. \\
\quad+\mathrm{E}_{\boldsymbol{\lambda}}\left[\int_{x_{c}+\epsilon}^{\lambda_{\max }} 1 d x, \Omega_{\boldsymbol{\lambda}}-A_{\boldsymbol{\lambda}}^{n}\right] \\
\left.+\mathrm{E}_{\boldsymbol{\lambda}}\left[\int_{x_{c}+\epsilon}^{\lambda_{\max }} \delta d x, A_{\boldsymbol{\lambda}}^{n}\right]\right\} \\
=\bar{r}\left\{\mathrm{E}_{\boldsymbol{\lambda}}\left[x_{c}+\epsilon\right]+\left(\lambda_{\max }-x_{c}-\epsilon\right)\left(1-\operatorname{Pr}\left(A_{\boldsymbol{\lambda}}^{n}\right)\right)\right. \\
\left.\quad+\delta\left(\lambda_{\max }-x_{c}-\epsilon\right) \operatorname{Pr}\left(A_{\boldsymbol{\lambda}}^{n}\right)\right\} \\
\rightarrow \quad \bar{r}\left\{x_{c}+\epsilon+\delta\left(\lambda_{\max }-x_{c}-\epsilon\right)\right\} .
\end{gathered}
$$

By taking $\epsilon$ and $\delta$ arbitrarily small, we have

$$
\lim I^{(n)} \leq \bar{r} x_{c} .
$$

The essential tool used to prove 8 is the twisted distribution [4]. This tool is the main tool in proving the lower bound of Cramer's theorem [4], a basic result of large deviations. However, there is a fundamental difference between the standard large deviation technique and our approach. While in Cramer's theorem one considers the sums of i.i.d. random variables, here we consider the sum of $\left(\lambda_{i}-x\right)\left|z_{i}\right|^{2}$, where the random variables are conditional independent but not identically distributed and the condition itself is a random vector. While the conditional independence requires us to discuss the statistics of $\sum\left(\lambda_{i}-x\right)\left|z_{i}\right|^{2}$ on the set $A_{\lambda}^{n}$, the non-identical distribution brings the major difficulty. That is, the twisted distribution may or may not be well-defined. To overcome this difficulty, we have to discuss two types of $x$ and define two types of twisted distributions respectively.

We define two types of $x$ and two types of twisted distributions as follows. Let $\alpha_{x}$ be the $\alpha$ such that $\bar{\psi}(x)=\psi\left(x, \alpha_{x}\right)$. The set of $x$ of the first type is defined by

$$
\mathcal{X}_{1} \triangleq\left\{x \in\left(\lambda_{t}^{-}, \lambda^{+}\right): \alpha_{x} \in\left(0, \frac{1}{x-\lambda_{t}^{-}}\right)\right\} .
$$

The set of $x$ of the second type is defined by

$$
\mathcal{X}_{2} \triangleq\left\{x \in\left(\lambda_{t}^{-}, \lambda^{+}\right): \alpha_{x}=\frac{1}{x-\lambda_{t}^{-}}\right\} .
$$

It can be proved that the $x_{c}$ in Theorem 1 is either in $\mathcal{X}_{1}$ or in $\mathcal{X}_{2}$. If $x \in \mathcal{X}_{1}$, then $\alpha_{x}<\frac{1}{x-\lambda_{t}^{-}}$and $\mathrm{E}_{\mathbf{z}}\left[e^{-\alpha \sum\left(\lambda_{i}-x\right)\left|z_{i}\right|^{2}}\right]$ is well defined on the set $A_{\lambda}^{n}$ with $\epsilon_{2}$ small enough. Then we are able to define a twisted distribution measure

$$
d \tilde{\mu}_{\mathbf{z}} \triangleq \frac{e^{-\alpha \sum\left(\lambda_{i}-x\right)\left|z_{i}\right|^{2}}}{\mathrm{E}_{\mathbf{z}}\left[e^{\left.-\alpha \sum\left(\lambda_{i}-x\right)\left|z_{i}\right|^{2}\right]}\right.} d \mu_{\mathbf{z}}
$$

where $d \mu_{\mathbf{z}}$ is the probability measure for the random vector $\mathbf{z}$. However, if $x \in \mathcal{X}_{2}, \mathrm{E}_{\mathbf{z}}\left[e^{-\alpha \sum\left(\lambda_{i}-x\right)\left|z_{i}\right|^{2}}\right]$ is not well defined on the set $A_{\lambda}^{n}$ no matter how small $\epsilon_{2}>0$ we choose. For this case, we have to define the twisted distribution in a "truncated" way. Define the $M$-truncated measure for $\mathbf{z}$ as

$$
d \mu_{\mathbf{z}}^{M}=\prod_{i=1}^{n} \mathbb{1}_{z_{i} \in[0, M]} d \mu_{\mathbf{z}}
$$

Then the $M$-truncated twisted distribution measure is defined by

$$
d \tilde{\mu}_{\mathbf{z}}^{M} \triangleq \frac{e^{-\alpha \sum\left(\lambda_{i}-x\right)\left|z_{i}\right|^{2}}}{\mathrm{E}_{\mu_{\mathbf{z}}^{M}}\left[e^{-\alpha \sum\left(\lambda_{i}-x\right)\left|z_{i}\right|^{2}}\right]} d \mu_{\mathbf{z}}^{M} .
$$

It can be verified that $d \tilde{\mu}_{\mathbf{z}}^{M}$ is always well defined on the set $A_{\lambda}^{n}$ for a finite $M>0$. In the proof of $[8$, we need to choose an $M$ sufficiently large.

With the twisted distributions, 8 can be proved. Here, we only outline the proof for $x_{c} \in \mathcal{X}_{1}$, the simpler case. Assume that $x_{c} \in \mathcal{X}_{1}$. For an $\epsilon>0$ small enough, let $x=x_{c}+\frac{\epsilon}{2}$. It 
can be proved that $x \in \mathcal{X}_{1}$ and $\bar{\psi}(x)=\psi\left(x, \alpha_{x}\right)<c \log 2$. For $\forall \delta_{1}>0$ and a $y>x=x_{c}+\frac{\epsilon}{2}$, it can be proved that

$$
F_{n}(y \mid \boldsymbol{\lambda}) \geq \operatorname{Pr}\left(\sum\left(\lambda_{i}-x\right)\left|z_{i}\right|^{2} \leq n \epsilon_{3}\right)\left(1-\delta_{1}\right)
$$

on the set $A_{\lambda}^{n}$ with small enough $\epsilon_{3}$ and large enough $n$. But for any given $\epsilon_{3}>0$, a further lower bound can be derived as follows.

$$
\begin{array}{ll} 
& \operatorname{Pr}\left(\sum\left(\lambda_{i}-x\right)\left|z_{i}\right|^{2} \leq n \epsilon_{3}\right) \\
\stackrel{(a)}{\geq} & \operatorname{Pr}\left(-\alpha_{x} \sum\left(\lambda_{i}-x\right)\left|z_{i}\right|^{2} \in n\left(-\delta_{2}, \delta_{2}\right)\right) \\
\stackrel{(b)}{\geq} & e^{-n \delta_{2}} \int_{B_{\mathbf{z}}} e^{-\alpha_{x} \sum\left(\lambda_{i}-x\right)\left|z_{i}\right|^{2}} d \mu_{\mathbf{z}} \\
\stackrel{(c)}{=} & e^{-n \delta_{2}} \mathrm{E}_{\mathbf{z}}\left[e^{-\alpha_{x} \sum\left(\lambda_{i}-x\right)\left|z_{i}\right|^{2}}\right] \int_{B_{\mathbf{z}}} d \tilde{\mu}_{\mathbf{z}} \\
\stackrel{(d)}{\geq} & \exp \left\{-n\left[\delta^{\prime}+\psi\left(x, \alpha_{x}\right)\right]\right\} P_{\tilde{\mu}_{\mathbf{z}}}\left(B_{\mathbf{z}}\right),
\end{array}
$$

on the set $A_{\lambda}^{n}$ with large enough $n$, where

(a) holds by choosing $\delta_{2}<\frac{\epsilon}{\alpha_{x}}$,

$$
\text { holds by defining }
$$

$$
B_{\mathbf{z}} \triangleq\left\{\mathbf{z}:-\alpha_{x} \sum\left(\lambda_{i}-x\right)\left|z_{i}\right|^{2} \in n\left(-\delta_{2}, \delta_{2}\right)\right\},
$$

(c) follows from the definition of the twisted distribution for $x \in \mathcal{X}_{1}$, and

(d) follows by letting $\delta^{\prime}=\epsilon_{1}+\delta_{2}$, where $P_{\tilde{\mu}_{\mathbf{z}}}\left(B_{\mathbf{z}}\right)$ is the probability of $B_{\mathbf{z}}$ under the twisted distribution.

We want to calculate $P_{\tilde{\mu}_{\mathbf{z}}}\left(B_{\mathbf{z}}\right)$. By studying the asymptotic behavior of $\frac{d}{d \alpha} \log \mathrm{E}_{\mathbf{z}}\left[e^{-\alpha_{x} \sum\left(\lambda_{i}-x\right)\left|z_{i}\right|^{2}}\right]$, it can be proved that for $\forall \delta^{\prime \prime}>0, P_{\tilde{\mu}_{\mathbf{z}}}\left(B_{\mathbf{z}}\right) \geq 1-\delta^{\prime \prime}$ on the set $A_{\lambda}^{n}$ with large enough $n$. Therefore, for $\forall \delta^{\prime \prime \prime}>0$,

$$
F_{n}(y \mid \boldsymbol{\lambda}) \geq e^{-n\left[\bar{\psi}(x)+\delta^{\prime}\right]}\left(1-\delta^{\prime \prime \prime}\right)
$$

on the set $A_{\lambda}^{n}$ with large enough $n$. Now we choose $\delta^{\prime}$ small enough such that $\bar{\psi}(x)+2 \delta^{\prime}<c \log 2$. Then it can be proved that, for $\forall \delta>0$,

$$
\begin{aligned}
& {\left[1-F_{n}(y \mid \boldsymbol{\lambda})\right]^{2^{c n}} } \\
\leq & \exp \left[-\left(1-\delta^{\prime \prime \prime}\right) e^{-n\left[\bar{\psi}(x)+\delta^{\prime}-c \log 2\right]}\right] \\
< & \delta
\end{aligned}
$$

on the set $A_{\lambda}^{n}$ with large enough $n$. Without loss of generality, we take $y=x_{c}+\epsilon>x=x_{c}+\frac{\epsilon}{2}$. Since $F_{n}(y \mid \boldsymbol{\lambda})$ is a nondecreasing function, we have uniform boundedness,

$$
\left[1-F_{n}(y \mid \boldsymbol{\lambda})\right]^{2^{c n}}<\delta \text { for all } y>x_{c}+\epsilon
$$

on the set $A_{\lambda}^{n}$ with large enough $n$. This is $\underline{8}$, what we want.

\section{Direction Matching Criterion}

In this section, we shall analyze the performance corresponding to the direction matching criterion in (2). Again, by letting $n, m$ and $R_{\mathrm{fb}}$ approach infinity simultaneously with fixed ratios, we derive the exact performance limit. The result is given in Theorem 2
Theorem 2: Following the definitions in Theorem 11 let $n$, $m$ and $R_{\mathrm{fb}}$ approach infinity simultaneously with fixed ratios $\tau, \bar{r}$ and $c$. For any $0<c<\infty$,

$$
\lim _{\left(n, m, R_{\mathrm{fb}}\right) \rightarrow \infty} I^{(n)}=\left\{\begin{array}{ll}
\lambda_{t}^{-}\left(1-2^{-c}\right)+\bar{\lambda} 2^{-c} & \text { if } n \leq m \\
x_{c} & \text { if } n>m
\end{array},\right.
$$

where $\bar{\lambda}=\frac{m}{n}, x_{c}<\frac{m}{n}$ satisfies $D\left(\mu_{\bar{r}} \| \mu_{x_{c}}\right)=c \log 2$ and $D\left(\mu_{\bar{r}} \| \mu_{x_{c}}\right) \triangleq \bar{r} \log \frac{\frac{n}{r}}{x_{c}}+(1-\bar{r}) \log \frac{1-\bar{r}}{1-x_{c}}$ is known as the relative entropy.

Remark 2: Elementary calculations show that the asymptotic average interference $\lim I$, as a function of $c$, converges to the average eigenvalue and the minimum eigenvalue as $c \rightarrow$ $0^{+}$and $c \rightarrow \infty$ respectively. These results are consistent with intuitions.

The proof of Theorem 2 is based on the observation that

$$
\begin{aligned}
I^{(n)}=\bar{r} & \sum_{i=1}^{n-d} \mathrm{E}_{\mathbf{U}}\left[\mathrm{E}_{\mathcal{B}}\left[\mathbf{s}_{1}^{\dagger} \mathbf{u}_{i} \mathbf{u}_{i}^{\dagger} \mathbf{s}_{1}\right]\right] \mathrm{E}_{\boldsymbol{\Lambda}}\left[\lambda_{i}\right] \\
& +\bar{r} \sum_{i=n-d+1}^{n} \mathrm{E}_{\mathbf{U}}\left[\mathrm{E}_{\mathcal{B}}\left[\mathbf{s}_{1}^{\dagger} \mathbf{u}_{i} \mathbf{u}_{i}^{\dagger} \mathbf{s}_{1}\right]\right] \mathrm{E}_{\boldsymbol{\Lambda}}\left[\lambda_{n}\right]
\end{aligned}
$$

where $\lambda_{1} \geq \cdots \geq \lambda_{n}$ are the singular values of $\frac{1}{r} \mathbf{H}_{n} \mathbf{H}_{n}^{\dagger}$ and $\mathbf{u}_{i}$ is the singular vector corresponding $\lambda_{i}$. For $n \leq m$ (full rank) case, $d=1$ with probability 1 . We select the signature $\mathbf{s}_{1}$ to match $\mathbf{u}_{n}$. The corresponding $\mathrm{E}_{\mathbf{U}}\left[\mathrm{E}_{\mathcal{B}}\left[\mathbf{s}_{1}^{\dagger} \mathbf{u}_{n} \mathbf{u}_{n}^{\dagger} \mathbf{s}_{1}\right]\right]$ can be calculated based on our previous results in the Grassmann manifold [5]. For $n>m$ (deficient rank) case, $d=n-m$ with probability 1 . We need to choose the signature $\mathbf{s}_{1}$ to match the plane generated by $\mathbf{U}_{d}=\left[\mathbf{u}_{n-d+1} \cdots \mathbf{u}_{n}\right]$. By large deviation technique, the corresponding $\mathrm{E}_{\mathbf{U}}\left[\mathrm{E}_{\mathcal{B}}\left[\mathbf{s}_{1}^{\dagger} \mathbf{U}_{d} \mathbf{U}_{d}^{\dagger} \mathbf{s}_{1}\right]\right]$ can be evaluated. The detailed derivation is given in [2].

\section{Simulations AND Discussion}

Fig. 11 shows the simulation results to demonstrate the asymptotic performance formulas (4) and (9) for both criteria. Fig. 1 a) and 1 b) are for $n \leq m$ (full rank) and $n>m$ (deficient rank) cases respectively. From the simulations, we can observe that the simulated average interference for both criteria (x markers for interference minimization and circles for direction matching) converges to the asymptotic results (the solid line for interference minimization and the dashed line for direction matching) as $n, m$ and $R_{\mathrm{fb}}$ approach infinity with fixed ratios. Simulations also show that direction matching is a sub-optimal criterion.

We also compare our formula with the bound in [1], denoted as SH bound in Fig 11 In [1], an asymptotic lower bound on the average interference is given for the interference minimization criterion. It is plotted as the dotted line in Fig. 11 Note that when $\tau=2$ and $n \leq m$, with infinite feedback rate $(c=\infty), I^{(n)}$ should converge to the minimum eigenvalue $(1-\sqrt{2})^{2} \approx 0.17$. The bound in [1] is below this value even when $c$ is relatively small ( $c \geq 2.5$ in Fig [1). Generally speaking, the bound in [1] under-estimates the interference while our asymptotic formula (4) gives the exact performance limit. 


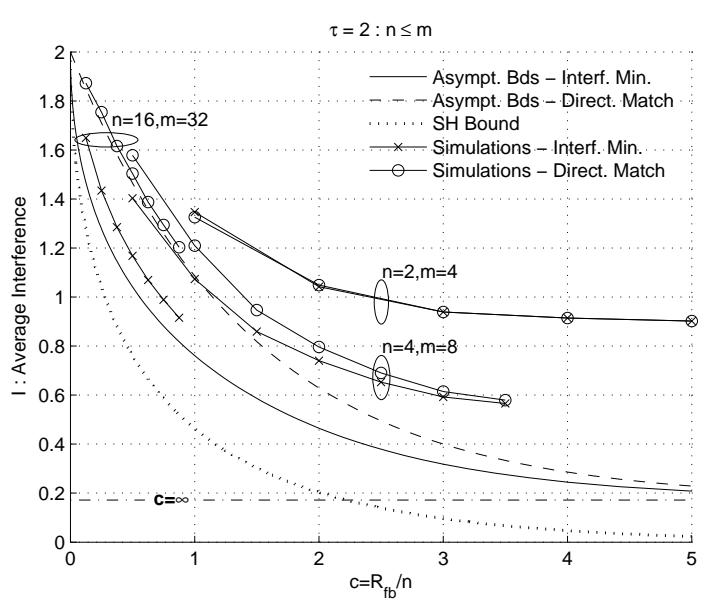

(a) Full Rank $(n \leq m)$ Case

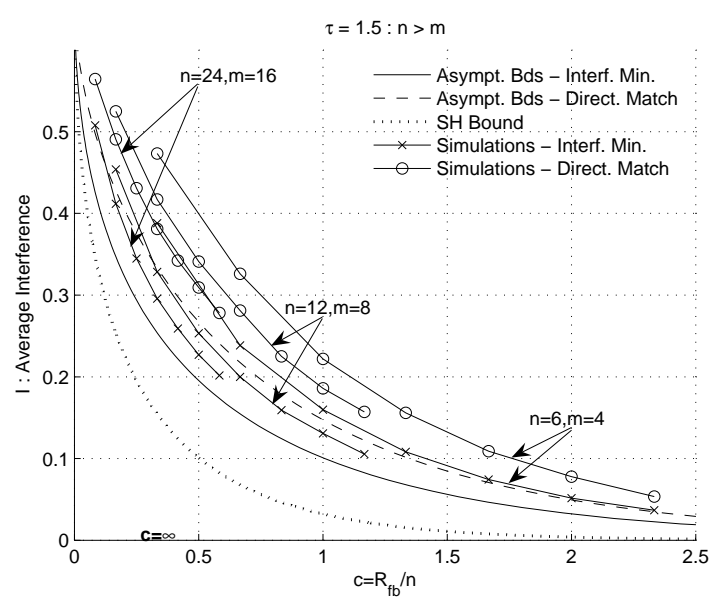

(b) Deficient Rank $(n>m)$ Case

Fig. 1. Simulations for both signature selection criteria

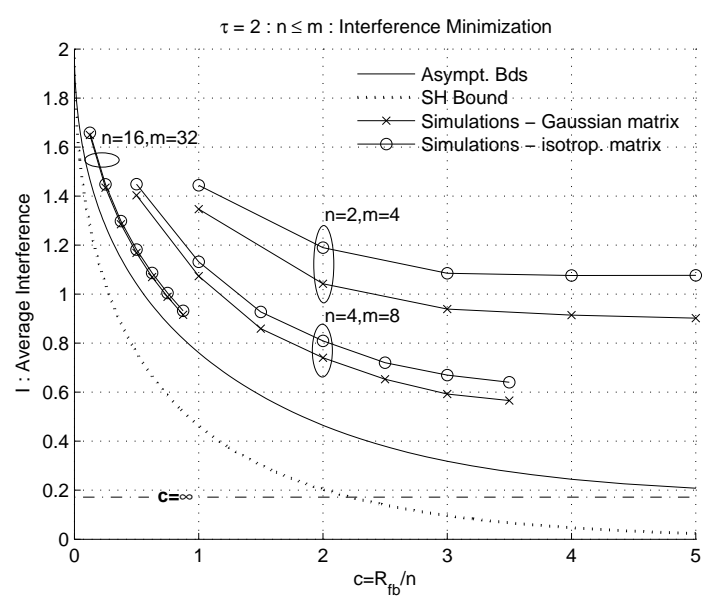

Fig. 2. Comparison of two types of interference matrices
As mentioned in the system model section, we assume that the interference matrix $\mathbf{S}$ has i.i.d. complex Gaussian entries with zero mean and variance $\frac{1}{n}$ for fair comparison, while it is more natural to assume that $\mathbf{S}$ has independent and isotropically distributed unitary complex columns. Fig 2 gives the difference between these two statistical assumptions, where the simulations are based on the interference minimization criterion. For small $n$ and $m$, these two different assumptions give two different results. However, as $n$ and $m$ increase, for example, $n=16$ and $m=32$, the difference becomes indistinguishable. Indeed, the asymptotic statistics of these two types of random matrices are identical. The results (4) and (9) are the exact asymptotic performance for both interference statistical assumptions.

\section{CONCLUSION}

In this paper, we quantify the average interference as a function of finite feedback rate for CDMA signature optimization problem. Two signature selection criteria, i.e., interference minimization and direction matching, are analyzed. By letting the processing gain, number of users and feedback bits approach infinity with fixed ratios, we derive the exact asymptotic formulas to calculate the average interference for both criteria respectively. The asymptotic results are valid for both the Gaussian interference matrix and the interference matrix with independent and isotropically distributed columns. Furthermore, the corresponding analysis can be extended to SINR performance evaluation for both match filter and linear MMSE receivers.

\section{REFERENCES}

[1] W. Santipach and M. Honig, "Signature optimization for CDMA with limited feedback," Information Theory, IEEE Transactions on, vol. 51, no. 10, pp. 3475-3492, 2005.

[2] W. Dai, Y. Liu, and B. Rider, "On the performance of CDMA signature optimization with finite rate feedback," In preparation for journal submission.

[3] R. J. Muirhead, Aspects of multivariate statistical theory. New York: John Wiley and Sons, 1982.

[4] A. Dembo and O. Zeitouni, Large Deviation Techniques in Decision, Simulation, and Estimation. Jones and Bartlett Publishers, 1992.

[5] W. Dai, Y. Liu, and B. Rider, "Quantization bounds on Grassmann manifolds of arbitrary dimensions and MIMO communications with feedback," in IEEE Global Telecommunications Conference (GLOBECOM), 2005. 